UWThPh-2003-37

December 19, 2003

\title{
Finite supersymmetry transformations
}

\author{
Nevena Ilieva ${ }^{a, c, *}$, Heide Narnhofer ${ }^{b}$ and Walter Thirring ${ }^{b, c}$ \\ ${ }^{a}$ Theory Division, CERN \\ ${ }^{b}$ Institut für Theoretische Physik, Universität Wien \\ ${ }^{c}$ Erwin Schrödinger International Institute for Mathematical Physics, Vienna
}

\begin{abstract}
We investigate simple examples of supersymmetry algebras with real and Grassmann parameters. Special attention is payed to the finite supertransformations and their probability interpretation. Furthermore we look for combinations of bosons and fermions which are invariant under supertransformations. These combinations correspond to states that are highly entangled.
\end{abstract}

* On leave from Institute for Nuclear Research and Nuclear Energy, Bulgarian Academy of Sciences, Boul.Tzarigradsko Chaussee 72, 1784 Sofia, Bulgaria 


\section{Introduction}

Supersymmetry is well understood and widely exploited in QFT and in models as a set of infinitesimal transformations generating some essential selection rules (see, e.g. [1]. The step towards obtaining the finite supertransformations is usually considered as unneccessary, thus placing SUSY in a distinguished position as compared with the other symmetries we know. On the other hand, already the simplest supersymmetric model SUSY QM [2], has a consistent physical interpretation in completely conventional means as it is equivalent to one particle and one spin, a system for which e.g. the probability interpretation perfectly holds. This observation motivates one to inquire how far is it possible to construct a realization of SUSY that avoids the introduction of Grassmann parameters and thus allows for a probability interpretation. Some efforts in this direction are due to Levine and Tomozawa [3] who tried doing this at the price of additional generators in the (extended) Lie algebra.

We choose another strategy, namely we consider a graded *-algebra $\mathcal{A}$ which is generated by some bosonic and fermionic operators and possibly some Grassmann or Clifford variables. As supertransformations we take ${ }^{*}$-automorphisms of $\mathcal{A}$ which do not respect the grading, thus mix bosons and fermions. The only other structural element we use is a one-parameter group of automorphisms of $\mathcal{A}$ (the time evolution) which commutes with the supertransformations. The emphasis of this paper is different from the one most commonly seen in the wast literature on this subject $\left(\sim 10^{4.5}\right.$ papers $)$ in the following respects:

(i) We consider the supersymmetry as a symmetry in the conventional understanding. Thus we do not stay on the Lie-algebraic level but consider finite supertransformations. Therefore for our operators the product is also defined and not only the commutator or the anticommutator;

(ii) We do not require that the time evolution is part of the Poincare-group which is represented by automorphism groups of $\mathcal{A}$;

(iii) We want to work with the standard probability interpretation of quantum mechanics. There a state over a ${ }^{*}$-algebra gives a probability distribution and a representation. The case II of Grassmann variables (defined in Sec.2) becomes then entirely strange. In the representation there are vectors of zero norm and all transition probabilities in this sector vanish.

In this context, two important questions to be answered are the following: what happens under such supersymmetry transformations with the states and which are the invariant structures. The former is crutial for the probability interpretation of the theory, for the latter, an example of an invariant state is certainly provided by the Fock vacuum. However, the interplay between supersymmetry and temperature and its consequences for the particle physics are less trivial and are still not matter of consense.

Some of these issues we could find only briefly discussed in the literature (see, e.g. $[1,4]$ ), others, up to our knowledge, have not yet received the due attention. In particular, 
the question whether the usual thermal state over one bosonic and one fermionic mode breaks the supersymmetry with Grassmann variables is confusing because of the seemingly contradictory results to be found in the literature. Girardello et al. [5] concluded that SUSY is broken because only the ground state is annihilated by the supercharges and the higher states are not. Though this statement is correct, van Hove calculated carefully all contributions to the change of the state to first order in the superparameter and showed that they cancel [6]. Thus he concluded that SUSY was not broken. Again this conclusion is incorrect, as we find that for the supercharges there is a change in the state (Sec. 6). Then came the sweeping proof of Buchholz and Ojima [7] that the KMS-properties and SUSY are incompatible even to first order. This proof seems impeccable since it just uses the fact that a supersymmetric Hamiltonian is positive but for a KMS-state its spectrum is $\mathbf{R}$. This appears in the thermodynamic limit. Here we concentrate on finitely many modes of fermions and bosons and therefore the spectrum is semibounded and discrete. Already on this level we will see that odd derivations violate the invariance of the thermal state.

Our result is that supertransformations can very well be constructed without the help of Grassman variables. Moreover, when Grassmann variables are involved, the supertransformations change the thermal state, while in the case of real variables they leave it invariant.

\section{$1 \quad *$-automorphisms and supertransformations}

The problems addressed in what follows are matters of principle and appear already in the simplest supersymmetric situation of one Bose- and one Fermi- mode. There we have the supersymmetry as a one-parameter group of transformations of the algebra $\mathcal{A}$ generated by the two creation and annihilation operators. In fact, we shall consider three different mixed Bose-Fermi algebras $-\mathcal{A}, \mathcal{A}_{\theta}$ and $\mathcal{A}_{C}$, defined as follows: ${ }^{1}$

I. $\mathcal{A}$ is the algebra generated by the operators $a, a^{\dagger}, b, b^{\dagger}$, satisfying CAR, resp. CCR

$$
\begin{aligned}
\left\{a, a^{\dagger}\right\} & =\left[b, b^{\dagger}\right]=1 \\
{[a, b] } & =\left[a, b^{\dagger}\right]=0 .
\end{aligned}
$$

II. The algebra $\mathcal{A}_{\theta}$ is the same $\mathcal{A}$, extended by a Grassmann variable $\theta$ so that

$$
\theta=\bar{\theta}, \quad \theta^{2}=\{\theta, a\}=[\theta, b]=0 .
$$

III. Furthermore we consider the case of Clifford variables where we have $\{\theta, \bar{\theta}\}=1$, the corresponding algebra being denoted by $\mathcal{A}_{C}$. Its odd elements contain odd powers of the fermionic operators $a, a^{\dagger}$.

\footnotetext{
${ }^{1}$ To be conform with the common notations in the supersymmetry literature we denote the hermitian conjugation by ${ }^{\dagger}$ but we keep the ${ }^{*}$-terminology for the algebraic considerations.
} 
Remark $\mathcal{A}$ and $\mathcal{A}_{C}$ are $C^{*}$-algebras, whereas $\mathcal{A}_{\theta}$ is not, since the $C^{*}$-condition $\|\bar{\theta} \theta\|=$ $\|\theta\|^{2}$ would imply $\|\theta\|=0$, therefore $\theta=0$. $\mathcal{A}_{\theta}$ is however a ${ }^{*}$-algebra and a Grassmann module; any of its elements can be written as $A+\theta B$ [8]. The "soul" $\theta B$ is a two-sided ideal of $\mathcal{A}_{\theta}$, the "body" $\mathcal{A}$ being the quotient algebra. The Hilbert-space representations are not faithful, in them the soul vanishes. In such a representation $e^{i s G_{\theta}}=1$ and the supertransformations become an illusion.

A unitary element $U, U^{\dagger}=U^{-1}$, creates a transformation of the algebra which preserves both the multiplicative and the ${ }^{*}$-structure. We are interested in one-parameter groups of supertransformations which preserve the total particle number $N=a^{\dagger} a+b^{\dagger} b=$ : $N_{f}+N_{b}$ but not the individual $N_{f}$ and $N_{b}$. The simplest generators for such transformations for the algebras under consideration are the hermitean elements

$$
\begin{array}{ll}
G=a b^{\dagger}+a^{\dagger} b, & \text { for } \mathcal{A} \\
G_{\theta}=-i \theta\left(a b^{\dagger}+a^{\dagger} b\right)=G_{\bar{\theta}}, & \text { for } \mathcal{A}_{\theta} \\
G_{\theta, \bar{\theta}}=-i\left(\theta a b^{\dagger}+\bar{\theta} a^{\dagger} b\right), & \text { for } \mathcal{A}_{C}
\end{array}
$$

Thus

$$
A \rightarrow A(s)=e^{i G s} A e^{-i G s}, \quad A \in \mathcal{A}
$$

gives a ${ }^{*}$-automorphism group of $\mathcal{A}$ and similarly for $\mathcal{A}_{\theta}$. From the definitions follows $G^{2}=N$ and $G_{\theta}^{2}=0$. Thus

$$
e^{i G s}=\cos \sqrt{N} s+\frac{i G}{\sqrt{N}} \sin \sqrt{N} s
$$

and

$$
e^{i G_{\theta} s}=1+\theta G s
$$

\section{Remarks}

1. Alternatively we could have considered $G_{\mathcal{A}}:=i\left(a^{\dagger} b-a b^{\dagger}\right)$ but this is equivalent to $G$ since the two are related by an even transformation commuting with the time evolution $e^{i N t}: G_{\mathcal{A}}=e^{i N_{f} \pi / 2} G e^{-i N_{f} \pi / 2}$. However we shall later find a time evolution which is still supersymmetric in the sense that it leaves $G$ invariant but no longer $G_{\mathcal{A}}$

2. As another alternative, one can consider the group generated by both $G$ and $G_{\mathcal{A}}$. This group is infinite-dimensional since $\left[G, G_{\mathcal{A}}\right]=2 i\left(b^{\dagger} b-a^{\dagger} a-2 a^{\dagger} a b^{\dagger} b\right)$ is not linearly expressible by these generators. In contradistinction, the corresponding generators with Grassmann parameters $-G_{\theta}$ and $G_{\mathcal{A} \theta}$, form a two-dimensional group;

3. The group generated by $G$ and $N$ is just the product of the two groups and thus isomorphic to $\mathbf{R}^{\mathbf{2}}$, in sharp contrast to the Lie superalgebra. The only trace on the level of finite transformations of the relation between $N$ and $G$ is the simple expression (1.5) for $e^{i G s}$. 
Thus we conclude that there are four different candidates for possible SUSY transformation of the three algebras:

Ia. In $\mathcal{A}$ one can generate a one-parameter group of automorphisms by

$$
A(s)=e^{i G s} A e^{-i G s}, \quad G=a^{\dagger} b+a b^{\dagger}, A \in \mathcal{A} ;
$$

In this spirit, for instance, the Jaynes-Cummings model has been investigated in [9].

Ib. In $\mathcal{A}$ an odd derivation generates a one-parameter group of linear transformations by

$$
A^{\prime}=\frac{d}{d s} A(s):=\delta A, \quad \delta A= \begin{cases}\{G, A\} & \text { for } A \text { odd } \\ i[G, A] & \text { for } A \text { even }\end{cases}
$$

where $A$ is the basis $(a, b)$ of $\mathcal{A}$ and $\delta$ is such that $\delta A^{*}=(\delta A)^{*}$. Thus one can express supertransformations infinitesimally without Grassmann or Clifford variables (see, e.g. $[7,10])$. We take the convention with the factors $i$ such that $\delta$ is compatible with conjugation, $\delta A^{*}=(\delta A)^{*}$. However, $\delta$ is not compatible with multiplication since only for even elements $b_{i}$ it satisfies the Leibnitz rule $\delta\left(b_{1} b_{2}\right)=\left(\delta b_{1}\right) b_{2}+b_{1} \delta b_{2}$. For odd elements $a_{i}$ we get $\delta(a b)=(\delta a) b+i a(\delta b)$ and $\delta\left(a_{1} a_{2}\right)=i\left(\delta a_{1}\right) a_{2}-i a_{1} \delta a_{2}$. Therefore it can be integrated to a one-parameter group of maps $\mathcal{A} \rightarrow \mathcal{A}$ which commute with the time evolution and respect the linear and ${ }^{*}$-structure of $\mathcal{A}$ but not its multiplicative structure. Note that for any element $A \in \mathcal{A}, \delta^{2} A=\delta \delta A=i\left[G^{2}, A\right]$, which gives its time derivative if $G^{2}=H$.

II. In $\mathcal{A}_{\theta}$ a one-parameter group of automorphisms is generated by

$$
A(s)=e^{i G_{\theta} s} A e^{-i G_{\theta} s}, \quad G_{\theta}=-i \theta G=G_{\theta}^{\dagger}, A \in \mathcal{A}_{\theta} ;
$$

III. In $\mathcal{A}_{C}$ the operator $G_{\theta, \bar{\theta}}=-i\left(\theta a b^{\dagger}+\bar{\theta} a^{\dagger} b\right)=G_{\theta, \bar{\theta}}^{\dagger}$ in turn generates a one-parameter automorphism group

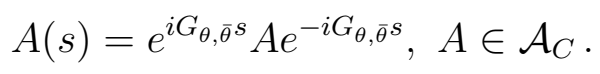

Such a transformation becomes of importance in the noncommutative supersymmetric models, which are defined by algebraic structure of this type (see, e.g. [11]).

Note that in the last two cases we are faced with automorphisms of the extended algebras which do not leave $\mathcal{A}$ as a set invariant.

\section{Finite supertransformation and differential char- acterization of the groups}

Ia. In order to obtain from Eq.(1.5) the finite supertransformations in $\mathcal{A}$ explicitly one has to make use of the relations

$$
\begin{gathered}
N a=a(N-1), \quad N b=b(N-1) \\
G a=b-a G, \quad G b=-a+b G
\end{gathered}
$$


to rearrange the products of

$$
a(s)=\left(\cos \sqrt{N} s+\frac{i G}{\sqrt{N}} \sin \sqrt{N} s\right) a\left(\cos \sqrt{N} s-\frac{i G}{\sqrt{N}} \sin \sqrt{N} s\right)
$$

and

$$
b(s)=\left(\cos \sqrt{N} s+\frac{i G}{\sqrt{N}} \sin \sqrt{N} s\right) b\left(\cos \sqrt{N} s-\frac{i G}{\sqrt{N}} \sin \sqrt{N} s\right) .
$$

This gives

$$
\begin{aligned}
a(s) & =\cos \sqrt{N} s \cos \sqrt{N+1} s a+\frac{\sin \sqrt{N} s \sin \sqrt{N+1} s}{\sqrt{N(N+1)}} a^{\dagger} b^{2} \\
& +i \frac{\sin \sqrt{N} s \cos \sqrt{N+1} s}{\sqrt{N}} a a^{\dagger} b-i \frac{\sin \sqrt{N+1} s \cos \sqrt{N} s}{\sqrt{N+1}} a^{\dagger} a b \\
b(s) & =\cos \sqrt{N} s \cos \sqrt{N+1} s b+\frac{\sin \sqrt{N} s \sin \sqrt{N+1} s}{\sqrt{N(N+1)}}\left(b^{2} b^{\dagger}-2 a a^{\dagger} b\right) \\
& +i \frac{\sin \sqrt{N} s \cos \sqrt{N+1} s}{\sqrt{N}}\left(a b^{\dagger} b+a^{\dagger} b^{2}\right) \\
& -i \frac{\sin \sqrt{N+1} s \cos \sqrt{N} s}{\sqrt{N+1}}\left(a b b^{\dagger}+a^{\dagger} b^{2}\right)
\end{aligned}
$$

with

$$
a \equiv a(0) \quad b \equiv b(0)
$$

Ib. For the simple algebra at hand one gets $\delta a=b$ and $\delta b=-i a$, which can be integrated to

$$
\begin{aligned}
& a(s)=a \cos s \sqrt{i}+(b / \sqrt{i}) \sin s \sqrt{i} \\
& b(s)=b \cos s \sqrt{i}-a \sqrt{i} \sin s \sqrt{i}
\end{aligned}
$$

II. The supertransformation in $\mathcal{A}_{\theta}$ is particularly simple:

$$
\begin{gathered}
a(s)=a+s \theta b, \quad a^{\dagger}(s)=a^{\dagger}-s \theta b^{\dagger} \\
b(s)=b-\theta s a, \quad b^{\dagger}(s)=b^{\dagger}+\theta s a^{\dagger} \\
\theta(s)=\theta=\bar{\theta}(s) .
\end{gathered}
$$

One readily verifies that this is a ${ }^{*}$-automorphism.

III. For $G_{\theta, \bar{\theta}}^{2}$ there is no simple expression and thus no way to get explicite expressions for $a(s)$ and $b(s)$.

Alternatively we could first look at the differential equation which determines the flow. With the notation $A^{\prime}=d / d s A(s)$ we have for the formal derivatives: 


$$
\begin{array}{ll}
\text { Ia. } & \left\{\begin{array}{l}
a^{\prime}=-i b+2 i G a \\
b^{\prime}=-i a
\end{array}\right. \\
\text { Ib. } & \begin{cases}a^{\prime}=b \\
b^{\prime}=-i a\end{cases} \\
\text { II. } \quad \begin{cases}a^{\prime}=\theta b, \\
b^{\prime}=-\theta a\end{cases} & \theta^{\prime}=0 \\
\text { III. } \quad \begin{cases}a^{\prime}=\bar{\theta} b, & \theta^{\prime}=-b a^{\dagger} \\
b^{\prime}=-\theta a, & \bar{\theta}^{\prime}=-a b^{\dagger}\end{cases}
\end{array}
$$

\section{Remarks:}

1. Compatibility with the product structure and therefore with canonical commutation or anticommutation relations (CCR, resp. CAR) requires:

( $\alpha) a^{\dagger \prime} a+a^{\dagger} a^{\prime}+a^{\prime} a^{\dagger}+a a^{\dagger \prime}=0$

( $\beta) b^{\prime} b^{\dagger}+b b^{\prime \dagger}-b^{\prime \dagger} b-b^{\dagger} b^{\prime}=0$

$(\gamma) a^{\prime} b+a b^{\prime}-b a^{\prime}-b^{\prime} a=0$

Whereas $(\beta)$ and $(\gamma)$ are always satisfied, $(\alpha)$ holds only in cases Ia, II, III.

2. In cases II, III the commutation relations require in addition:

( $\alpha) \quad \theta^{\prime} a+\theta a^{\prime}+a^{\prime} \theta+a \theta^{\prime}=0$

(в) $\theta^{\prime} \bar{\theta}+\theta \bar{\theta}^{\prime}+\bar{\theta}^{\prime} \theta+\bar{\theta} \theta^{\prime}=0$.

These conditions are satisfied only in case II. Thus to say that the $\theta$ 's are just anticommutative numbers and are not changed by the transformation leads to inconsistencies.

To integrate the differential equations poses different problems in the four cases, although this way we could obtain again the finite transformations, Eqs.(2.1)-(2.4).

\section{Representations}

A representation $\pi$ is an isomorphism of the algebra with an operator algebra in a Hilbert space, a ${ }^{*}$-representation is a ${ }^{*}$-isomorphism, i.e. $\pi(A B)=\pi(A) \pi(B), \pi\left(A^{*}\right)=\pi(A)^{*}$. Thus for $\mathcal{A}_{\theta}$ we can only have representations and not ${ }^{*}$-representations since operator algebras are $C^{*}$-algebras. The GNS-construction leads at first to an inner-product space which contains zero-norm vectors created by the soul. Passing to the Hilbert space by quotioning them out we are left only with the body, the soul-ideal being represented by 
zero. But for a physical interpretation this procedure is unavoidable since the results of measurements are real and not Grassmann numbers. This seemingly purely mathematical distinction will have the consequence that in a representation the transformation in $\mathcal{A}_{\theta}$ has no probability interpretation. It will not give transition probabilities.

An obvious representation of $\mathcal{A}$ is given by a quantum particle with coordinates $(x, p)$ and one spin [2]:

$$
a=\frac{\sigma_{x}-i \sigma_{y}}{2}, \quad b=\frac{x+i p}{\sqrt{2}} .
$$

For $\mathcal{A}_{\theta}$ we have to use a second spin, described by Pauli-matrices $\tau_{k}$ and set $\theta=\sigma_{3}\left(\tau_{x}-\right.$ $\left.i \tau_{y}\right) / 2$. The usual Fock representation $\pi_{F}$ based on a "vacuum" $|0\rangle$ with $a|0\rangle=b|0\rangle=0$ appears to be the most convenient framework for our considerations. In $\pi_{F}$ an orthogonal basis is given by

$$
\left|n_{f}, n_{b}, n_{g}\right\rangle=\left(a^{\dagger}\right)^{n_{f}} \frac{\left(b^{\dagger}\right)^{n_{b}}}{\sqrt{n_{b} !}} \theta^{n_{g}}|0\rangle, \quad n_{f}, n_{g}=0,1, n_{b}=0,1,2, \ldots
$$

For $\mathcal{A}$ we have $n_{g}=0$, for $\mathcal{A}_{\theta}$ we have $\theta|0\rangle \neq 0$ but $\theta^{2}|0\rangle=0$. The action of $G$ is now rather simple

$$
\begin{aligned}
G\left|0, n_{b}\right\rangle & =\sqrt{n_{b}}\left|1, n_{b}-1\right\rangle \\
G\left|1, n_{b}\right\rangle & =\sqrt{n_{b}+1}\left|0, n_{b}+1\right\rangle,
\end{aligned}
$$

thus

$$
\begin{aligned}
e^{i G s}\left|0, n_{b}\right\rangle & =\cos \sqrt{n}_{b} s\left|0, n_{b}\right\rangle+i \sin \sqrt{n}_{b} s\left|1, n_{b}-1\right\rangle \\
e^{i G s}\left|1, n_{b}\right\rangle & =\cos \sqrt{n_{b}+1} s\left|1, n_{b}\right\rangle+i \sin \sqrt{n_{b}+1} s\left|0, n_{b}+1\right\rangle .
\end{aligned}
$$

The action of $e^{i G_{\theta} s}$ is even simpler

$$
\begin{aligned}
e^{i G_{\theta} s}\left|0, n_{b}, 0\right\rangle & =\left|0, n_{b}, 0\right\rangle-s \sqrt{n}_{b}\left|1, n_{b}-1,1\right\rangle \\
e^{i G_{\theta} s}\left|1, n_{b}, 0\right\rangle & =\left|1, n_{b}, 0\right\rangle-s \sqrt{n_{b}+1}\left|0, n_{b}+1,1\right\rangle \\
e^{i G_{\theta} s}\left|n_{f}, n_{b}, 1\right\rangle & =\left|n_{f}, n_{b}, 1\right\rangle .
\end{aligned}
$$

Once again, for $\mathcal{A}_{\theta} \pi_{F}$ is only a representation but cannot be a ${ }^{*}$-representation. As a consequence only $e^{i G s}$ but not $e^{i G_{\theta} s}$ has a physical interpretation. $e^{i G s}$ changes a boson into a fermion or vice versa. It does this with a probability $\sin ^{2} \sqrt{n_{b}} s$, resp. $\sin ^{2} \sqrt{n_{b}+1} s$, whereas it leaves the state unchanged with $\cos ^{2}$ probability. On the contrary, $e^{i G_{\theta} s}$ does nothing for $n_{g}=1$ and for $n_{g}=0$ it leaves the state unchanged with weight 1 and changes $n_{g}$ and bosons into fermions (or vice versa) with a weight $n_{b} s^{2}$, resp. $\left(n_{b}+1\right) s^{2}$, times $\| \theta|\rangle \|^{2}$ which is zero. Clearly, these weights should not be interpreted as probabilities and we are forced to conclude that the supertransformation in $\mathcal{A}_{\theta}$ is only an illusion, in contradistinction to the supertransformation in $\mathcal{A}$.

If we give up the hermiticity of $\theta$, that is $\theta$ is no longer hermitian but instead $\{\theta, \bar{\theta}\}=1$, still keeping the anticommutativity, the algebra $\mathcal{A}_{\theta}=\{a, b, \theta\}$ becomes the $C^{*}$-algebra 
$\mathcal{A}_{C}$ and we have a Fock ${ }^{*}$-representation $\pi_{F}$. It is based on the vacuum $|0\rangle$, which is annihilated by $\{a, b, \theta\}$. An orthogonal basis is given by

$$
\left|n_{f}, n_{g}, n_{b}\right\rangle=\left(a^{\dagger}\right)^{n_{f}}(\bar{\theta})^{n_{g}} \frac{\left(b^{\dagger}\right)^{n_{b}}}{\sqrt{n_{b} !}}|0\rangle, \quad n_{f}, n_{g}=0,1 ; n_{b}=0,1,2, \ldots
$$

We restrict ourselves to inspect the unitary implementer $e^{i G s}$ of the supertransformations. The previous $G$ generalises to $G_{\theta, \bar{\theta}}=\theta a b^{\dagger}+b a^{\dagger} \bar{\theta}$ and $G_{\theta, \bar{\theta}}^{2}$ is a bit more complicated, $G_{\theta, \bar{\theta}}^{2}=N_{b}\left(1-N_{f}\right)\left(1-N_{g}\right)+N_{f} N_{g}\left(1+N_{b}\right), N_{g}=\bar{\theta} \theta, G^{2}=N / 2$ for $N_{g}=1 / 2$. Still it is diagonal in $\pi_{F}$ and there is nothing wrong with the expansion (1.5), with $N$ replaced with $G^{2}$. To work it out we need the action of $G_{\theta, \bar{\theta}}$ :

$$
\begin{aligned}
G_{\theta, \bar{\theta}}\left|0,0, n_{b}\right\rangle & =\sqrt{n}_{b}\left|1,1, n_{b}-1\right\rangle \\
G_{\theta, \bar{\theta}}\left|1,1, n_{b}\right\rangle & =\sqrt{n_{b}+1}\left|0,0, n_{b}+1\right\rangle
\end{aligned}
$$

Therefore

$$
\begin{aligned}
& e^{i G_{\theta, \bar{\theta}^{s}}}\left|0,0, n_{b}\right\rangle=\cos \sqrt{n}_{b} s\left|0,0, n_{b}\right\rangle+i \sin \sqrt{n}_{b} s\left|1,1, n_{b}-1\right\rangle \\
& e^{i G_{\theta, \bar{\theta}^{s}}}\left|1,1, n_{b}\right\rangle=\cos \sqrt{n_{b}+1} s\left|1,1, n_{b}\right\rangle+i \sin \sqrt{n_{b}+1} s\left|0,0, n_{b}+1\right\rangle .
\end{aligned}
$$

Here we are dealing again with a $C^{*}$-algebra and a ${ }^{*}$ - representation, so the transition probabilities add up to unity. In fact they are identical to the ones we found in $\mathcal{A}, \theta$ acts like a fermion ("spurion") attached to the original one and does nothing exceptional. Therefore we should honestly declare that we have two fermions and restore the symmetry between fermions and bosons. As a side remark we shall show that going to a finite number of bosonic and fermionic modes changes in the Fock representation very little.

\section{Some generalizations}

\subsection{The $N$-fermion/ $N$-boson system}

The $N$-fermion/ $N$-boson system is defined through the algebra $\mathcal{A}=\left\{a_{i}, b_{j}\right\}, i, j, k, \ldots=$ $1,2, \ldots, N$, with the usual rules

$$
\begin{aligned}
& \left\{a_{i}, a_{k}^{\dagger}\right\}=\delta_{i k}=\left[b_{i}, b_{k}^{\dagger}\right] \\
& \left\{a_{i}, a_{k}\right\}=\left[b_{i}, b_{k}\right]=\left[a_{i}, b_{k}\right]=\left[a_{i}, b_{k}^{\dagger}\right]=0 .
\end{aligned}
$$

We go straight to the question of the supertransformation,

$$
G=\sum_{i=1}^{n} k_{i}\left(a_{i} b_{i}^{\dagger}+a_{i}^{\dagger} b_{i}\right) .
$$

In $G^{2}$ the quartic terms again reduce to quadratic expression

$$
G^{2}=\sum_{i=1}^{n} k_{i}^{2}\left(a_{i}^{\dagger} a_{i}+b_{i}^{\dagger} b_{i}\right)=: H .
$$


We call it $H$ since it looks like a popular Hamiltonian. In $H$ the terms with different $i$ commute, in $G$ they do not. As a consequence, $e^{i G s} \neq \otimes_{k=1}^{N} e^{i G_{k} s}$, but in the Fock representation it is still managable. At the risk of boring the experts we give bellow the relevant expressions for the two-boson/two-fermion system $(N=2)$ explicitly. In this case, in the orthogonal basis

$$
\left|n_{f_{i}}, n_{b_{j}}\right\rangle=\left(a_{1}^{\dagger}\right)^{n_{f_{1}}}\left(a_{2}^{\dagger}\right)^{n_{f_{2}}} \frac{\left(b_{1}^{\dagger}\right)^{n_{b_{1}}\left(b_{2}^{\dagger}\right)^{n_{b_{2}}}}}{\sqrt{n_{b_{1}} ! n_{b_{2}} !}}|0\rangle, \quad i, j=1,2
$$

$G$ acts as

$$
\begin{aligned}
G\left|0,0, n_{b_{1}}, n_{b_{2}}\right\rangle & =k_{1} \sqrt{n_{b_{1}}}\left|1,0, n_{b_{1}}-1, n_{b_{2}}\right\rangle+k_{2} \sqrt{n_{b_{2}}}\left|0,1, n_{b_{1}}, n_{b_{2}}-1\right\rangle \\
G\left|1,0, n_{b_{1}}, n_{b_{2}}\right\rangle & =k_{1} \sqrt{n_{b_{1}}+1}\left|0,0, n_{b_{1}}+1, n_{b_{2}}\right\rangle+k_{2} \sqrt{n_{b_{2}}}\left|1,1, n_{b_{1}}, n_{b_{2}}-1\right\rangle \\
G\left|0,1, n_{b_{1}}, n_{b_{2}}\right\rangle & =k_{1} \sqrt{n_{b_{1}}}\left|1,1, n_{b_{1}}, n_{b_{2}}\right\rangle+k_{2} \sqrt{n_{b_{2}}+1}\left|0,0, n_{b_{1}}, n_{b_{2}}+1\right\rangle \\
G\left|1,1, n_{b_{1}}, n_{b_{2}}\right\rangle & =k_{1} \sqrt{n_{b_{1}}+1}\left|0,1, n_{b_{1}}+1, n_{b_{2}}\right\rangle+k_{2} \sqrt{n_{b_{2}}+1}\left|1,0, n_{b_{1}}, n_{b_{2}}+1\right\rangle
\end{aligned}
$$

from which we calculate the unitary action of $e^{i G s}=\cos \sqrt{H} s+i \frac{G}{\sqrt{H}} \sin \sqrt{H} s$ to be

$$
\begin{gathered}
e^{i G s}\left|0,0, n_{b_{1}}, n_{b_{2}}\right\rangle=\cos s \sqrt{k_{1}^{2} n_{b_{1}}+k_{2}^{2} n_{b_{2}}}\left|0,0, n_{b_{1}}, n_{b_{2}}\right\rangle+ \\
i \frac{\sin s \sqrt{k_{1}^{2} n_{b_{1}}+k_{2}^{2} n_{b_{2}}}}{\sqrt{k_{1}^{2} n_{b_{1}}+k_{2}^{2} n_{b_{2}}}}\left(k_{1} \sqrt{n_{b_{1}}}\left|1,0, n_{b_{1}}-1, n_{b_{2}}\right\rangle+k_{2} \sqrt{n_{b_{2}}} \mid 0,1, n_{b_{1}}, n_{b_{2}}-1\right) \\
e^{i G s}\left|1,0, n_{b_{1}}, n_{b_{2}}\right\rangle=\cos s \sqrt{k_{1}^{2}\left(n_{b_{1}}+1\right)+k_{2}^{2} n_{b_{2}}}\left|1,0, n_{b_{1}}, n_{b_{2}}\right\rangle+ \\
i \frac{\sin s \sqrt{k_{1}^{2}\left(n_{b_{1}}+1\right)+k_{2}^{2} n_{b_{2}}}}{\sqrt{k_{1}^{2}\left(n_{b_{1}}+1\right)+k_{2}^{2} n_{b_{2}}}}\left(k_{1} \sqrt{n_{b_{1}}+1}\left|0,0, n_{b_{1}}+1, n_{b_{2}}\right\rangle+k_{2} \sqrt{n_{b_{2}}} \mid 1,1, n_{b_{1}}, n_{b_{2}}-1\right)
\end{gathered}
$$

Note that the transition probabilities for the three outcomes add up to unity.

In the general case of $N$ modes the orthogonal basis is given by

$$
\left|\left\{n_{j}\right\},\left\{m_{j}\right\}\right\rangle=\prod_{i=1}^{N}\left(a_{i}^{\dagger}\right)^{n_{i}}\left(b_{i}^{\dagger}\right)^{m_{i}}|0\rangle, \quad H\left|\left\{n_{j}\right\},\left\{m_{j}\right\}\right\rangle=E\left|\left\{n_{j}\right\},\left\{m_{j}\right\}\right\rangle
$$

with

$$
\|\left|\left\{n_{j}\right\},\left\{m_{j}\right\}\right\rangle \|=1 \quad \text { if } n_{j}=0,1 ; m_{k}=0,1,2, \ldots
$$

The action of $G$ and of the unitary transformation it implements, correspondingly become

$$
\begin{aligned}
G\left|\left\{n_{j}\right\},\left\{m_{j}\right\}\right\rangle & =\sum_{i=1}^{N} k_{i}\left(\sqrt{m_{i}+1}\left|n_{1}, \ldots, n_{i}-1, \ldots, n_{N}, m_{1}, \ldots, m_{i}+1, \ldots, m_{N}\right\rangle\right. \\
& \left.+\sqrt{m_{i}}\left|n_{1}, \ldots, n_{i}+1, \ldots, n_{N}, m_{1}, \ldots, m_{i}-1, \ldots, m_{N}\right\rangle\right)
\end{aligned}
$$




$$
\begin{aligned}
e^{i G s}\left|\left\{n_{j}\right\},\left\{m_{j}\right\}\right\rangle & =\cos \sqrt{E} s\left|\left\{n_{j}\right\},\left\{m_{j}\right\}\right\rangle+i \sin \sqrt{E} s \sum_{i=1}^{N} k_{i} / \sqrt{E} \\
\times & \left(\sqrt{m_{i}+1}\left|n_{1}, \ldots, n_{i}-1, \ldots, n_{N}, m_{1}, \ldots, m_{i}+1, \ldots, m_{N}\right\rangle\right. \\
& \left.+\sqrt{m}_{i}\left|n_{1}, \ldots, n_{i}+1, \ldots, n_{N}, m_{1}, \ldots, m_{i}-1, \ldots, m_{N}\right\rangle\right)
\end{aligned}
$$

Note that

$$
\|\|\rangle \|^{2}=\cos ^{2} \sqrt{E} s+\sin ^{2} \sqrt{E} s \sum_{i=1}^{N} k_{i}^{2} \times\left\{\begin{array}{cll}
\left(m_{i}+1\right) & \text { if } & n_{i}=1 \\
m_{i} & \text { if } & n_{i}=0
\end{array}\right\}=1 .
$$

\subsection{The poorman's Wess-Zumino model}

As a next generalization and a step towards the field-theory setting let us consider a model of one Bose- and one Fermi- mode, but with an interaction introduced through the following modification of the supercharge:

$$
\begin{gathered}
G=\tilde{Q}+\tilde{Q}^{\dagger} \\
Q^{\dagger}=a^{\dagger} b \rightarrow \tilde{Q}^{\dagger}=a^{\dagger}\left(b+g b^{\dagger} b\right) \\
Q=b^{\dagger} a \rightarrow \quad
\end{gathered}
$$

with $g$ real. This is nothing else but a prototype of the Wess-Zumino model [12] and we have been dealing so far with its free-theory limit $(g=0)$. Such operators on loop space have been considered in [13].

Again, the Hamiltonian is given by

$$
H_{g}=G^{2}=\left\{Q, Q^{\dagger}\right\}
$$

that is

$$
H_{g}=H_{0}+g H_{0}\left(b+b^{+}\right)-g b^{\dagger}+g^{2}\left(b^{\dagger} b\right)^{2}
$$

with $H_{0}=N$. Expansions (1.5), (1.6) still hold (because of Eq.(4.8)), as well as the conservation of the supercharge $G,[G, H]=0$.

There are many possibilities for the supercharges and with (4.8) we can always generate a time evolution commuting with the supertransformations. However, already in this simple model it turns out that these supertransformations with different charges generate an infinite-dimensional algebra contrary to the free case [14] which appears to be in this context a lucky exception.

\section{$5 \quad$ Supersymmetric quasiparticles}

The transformation (2.1), (2.2) mixes $a$ and $b$ in a rather complicated manner and the question arises whether a special combination $A$ is left intact so that $e^{i G s} A e^{-i G s}$ produces 
only a phase factor $e^{i \gamma s} A$. This means that the commutator with $G$ should reproduce $A$. For a polynomial in $a$ and $b$ this does not happen, commuting with $G$ keeps increasing the degree of the polynomial in $b$. However, for a nonpolynomial function $f\left(b^{\dagger} b\right)$ this is not necessarily so and we shall show now that even a simple choice allows to get for the transformed $A^{\prime}$ a phase factor with $\gamma= \pm 1$. We just take $f$ real, continuous and $f(x)=0 \quad \forall x \geq 1$. Then $b f\left(b^{\dagger} b\right)=f\left(b^{\dagger} b+1\right) b$ since $b\left(b^{\dagger} b\right)^{n}=\left(b^{\dagger} b+1\right)^{n} b \quad \forall n \in N$. But since $b^{\dagger} b \geq 0, f\left(b^{\dagger} b+1\right)=0$ and $b f\left(b^{\dagger} b\right)=f\left(b^{\dagger} b\right) b^{\dagger}=0$. Denoting $a a^{\dagger} f\left(b^{\dagger} b\right)$ by $P_{0}$, $a P_{0}=P_{0} a^{\dagger}=0$, we claim that

$$
\left[A_{ \pm}, G\right]=\mp A_{ \pm}, \quad \text { where } A_{ \pm}:=P_{0}(a \mp b) .
$$

In the Fock representation $P_{0}$ is the projection onto the vacuum.

Proof:

$$
\begin{aligned}
& G A_{ \pm}=\left(a b^{\dagger}+a^{\dagger} b\right) P_{0}(a \mp b)=0 \\
& b b^{\dagger}=1+b^{\dagger} b \quad \Rightarrow \quad P_{0} b b^{\dagger}=P_{0} a a^{\dagger}=P_{0} \\
& A_{ \pm} G=P_{0}(a \mp b)\left(a b^{\dagger}+a^{\dagger} b\right)=P_{0}\left(\mp b b^{\dagger} a+b\right)=\mp A_{ \pm} .
\end{aligned}
$$

Conclusion:

$$
A_{ \pm}(s)=e^{i s G} A_{ \pm} e^{-i s G}=e^{ \pm i s} A_{ \pm}, \text {thus } A_{ \pm}^{\dagger} A_{ \pm} \text {is invariant }
$$

Question: Is the bastard created by $A_{ \pm}$(the "susino") a boson or a fermion?

Answer: Though $\left(A_{ \pm}(s)\right)^{2}=0$, neither $\left[A_{ \pm}, A_{ \pm}^{\dagger}\right]$ nor $\left\{A_{ \pm}, A_{ \pm}^{\dagger}\right\}$ equals 1. $A_{ \pm}$correspond to elementary SUSY-excitations and by combining them we can construct the invariants $A_{ \pm} A_{ \pm}^{\dagger}$ and SUSY excitons $A_{ \pm} A_{\mp}^{\dagger}$ which have a phase factor $\gamma= \pm 2$.

\section{Remarks:}

1. Under the time evolution with $H=G^{2}$ the susinos $A_{ \pm}$evolve like the bosons or the fermions, $A_{ \pm}(t)=e^{i t} A_{ \pm}$, but the situation could be as in the $K^{0}-\overline{K^{0}}$ system: by a small perturbation neither the boson nor the fermion are time-invariant but only the susinos. Consider $H_{\alpha}=H+\alpha G+\alpha^{2} / 4=(G+\alpha / 2)^{2}$. Under its time evolution neither $a$ nor $b$ but only $A_{ \pm}$changes just by a phase factor. Thus in a perfectly supersymmetric situation physics may become quite unusual;

2. Under the supertransformation generated by $G_{\mathcal{A}}, A_{ \pm}$ocsillate rigidly

$$
\left[A_{ \pm}, G_{\mathcal{A}}\right]= \pm i A_{\mp} .
$$

More explicitly, with $A_{ \pm}(s)=e^{i s G_{\mathcal{A}}} A_{ \pm} e^{-i s G_{\mathcal{A}}}, A^{\prime}=d / d s A(s)$, we get $A_{+}^{\prime}=A_{-}$, $A_{-}^{\prime}=-A_{+}$and therefore the oscillations

$$
\begin{aligned}
& A_{+}(r)=A_{+}(0) \cos r+A_{-}(0) \sin r \\
& A_{-}(r)=-A_{+}(0) \sin r+A_{-}(0) \cos r
\end{aligned}
$$


3. $A_{ \pm}$can be generalized to

$$
A_{(m, n) \pm}=\left(a^{\dagger} b^{\dagger^{m-1}}+b^{\dagger^{m}}\right) P_{0}\left(a^{n} b^{n-1}+b^{n}\right)
$$

with the properties

$$
\begin{gathered}
\left(A_{(m, n) \pm}\right)^{\dagger}=A_{(n, m) \pm}, \quad A_{(m, n) \pm} A_{(n, r) \pm}=n A_{(m, r) \pm} \\
e^{i s G} A_{(m, n) \pm} e^{-i s G}=e^{ \pm i s(\sqrt{n}-\sqrt{m})} A_{(m, n) \pm} .
\end{gathered}
$$

It can be interpreted as absorbing $n$ and creating $m$ susinos.

4. For the evolution (2.3) even a linear combination of $a$ and $b$ changes only by a common factor, there we have

$$
a(s) / \sqrt{i}+b(s)=e^{t \sqrt{i}}(a / \sqrt{i}+b) ;
$$

5. The other two supertransformations mix in some $\theta$ 's and do not leave a function of $a$ and $b$ only invariant.

The unitaries $e^{i s G}$ have eigenvalues $e^{ \pm i s \sqrt{n}}$, for $n=0$ the eigenvector is the vacuum $|0\rangle$ and for $n=1$ they are $A_{ \pm}^{\dagger}|0\rangle$. For arbitrary $n$ their properties are described by the following lemma which relates two main streams of contemporary physics:

Lemma: Except for $n=0$, in all other eigenvectors of the super transformation $e^{i s G}$ the bosons and the fermions of the one-boson/one-fermion system are maximally entangled.

The Hilbert space of our system is the tensor product of the fermionic and bosonic Hilbert spaces, $\mathcal{H}=\mathcal{H}_{F} \otimes \mathcal{H}_{B}$ and vectors which are not of the product form are called entangled, i.e. the correlations they carry are of quantum and not of classical origin. A convenient measure of the entanglement of a vector |\rangle in $\mathcal{H}$ is the entropy of the fermionic density matrix $\rho_{F}=-\operatorname{Tr}_{\mathcal{H}_{\mathrm{B}}}|\rangle\langle|, \operatorname{Tr}_{\mathcal{H}_{\mathrm{B}}}$ being the partial trace in $\mathcal{H}_{B}$, namely

$$
E=-\operatorname{Tr}_{\mathcal{H}_{\mathrm{F}}} \rho_{\mathrm{F}} \ln \rho_{\mathrm{F}} \leq \ln 2 .
$$

However $\rho_{B}$, the state reduced to the bosons, has the same entropy as $\rho_{F}$. Fermions are thereby not prefered to bosons.

Proof:

One verifies (compare Remark 3 above)

$$
\left(a^{\dagger} b+b^{\dagger} a\right)(|1, n-1\rangle \pm|0, n\rangle)= \pm \sqrt{n}(|1, n-1\rangle \pm|0, n\rangle),
$$

thus

$$
e^{i s G}(|1, n-1\rangle \pm|0, n\rangle)=e^{ \pm i s \sqrt{n}}(|1, n-1\rangle \pm|0, n\rangle) .
$$


Calculating the fermionic density matrix $\rho_{F}$ we find that in all cases it corresponds to the tracial state, $\rho_{F}=1 / 2$ and thus $E=\ln 2$ for any $n$. Since the transformation with $e^{i s G_{\mathcal{A}}}$ is unitarily equivalent to the one with $e^{i s G}$ by a unitary that belongs to the Fermi subalgebra this does not change the entanglement and the above statement holds also for its eigenvectors.

The generalization to two modes is straightforward but the situation there is somewhat different. With the notations of Section $4.1, k_{1}=1, k_{2}=k$, the supercharge and the Hamiltonian become

$$
\begin{aligned}
& G=a_{1}^{\dagger} b_{1}+a_{1} b_{1}^{\dagger}+k\left(a_{2}^{\dagger} b_{2}+a_{2} b_{2}^{\dagger}\right) \\
& H=G^{2}=a_{1}^{\dagger} a_{1}+b_{1}^{\dagger} b_{1}+k^{2}\left(a_{2}^{\dagger} a_{2}+b_{2}^{\dagger} b_{2}\right)
\end{aligned}
$$

The eigenvalues of $H$ are $n_{f_{1}}+n_{b_{1}}+k^{2}\left(n_{f_{2}}+n_{b_{2}}\right)$, correspondingly those of $G$ are $\pm \sqrt{n_{f_{1}}+n_{b_{1}}+k^{2}\left(n_{f_{2}}+n_{b_{2}}\right)}$. In general, the eigenvectors of $H$ are four-fold degenerate (those of $G$ - resp. two-fold degenerate), except the ground state (the vacuum $|0\rangle:=$ $|0,0,0,0\rangle$ ), which is not degenerate, and the states with $n_{1}=0$ or $n_{2}=0$ (only one mode occupied), which are two-fold degenerate. We shall use the following basis in the $H$-space:

$$
\begin{aligned}
& \psi_{1}=\left|1, n_{b_{1}}, 1, n_{b_{2}}\right\rangle \\
& \psi_{2}=\left|1, n_{b_{1}}, 0, n_{b_{2}}+1\right\rangle \\
& \psi_{3}=\left|0, n_{b_{1}}+1,1, n_{b_{2}}\right\rangle \\
& \psi_{4}=\left|0, n_{b_{1}}+1,0, n_{b_{2}}+1\right\rangle
\end{aligned}
$$

Any eigenvector $\psi$ of $H$ with eigenvalue $1+n_{b_{1}}+k^{2}\left(1+n_{b_{2}}\right)$ can be written as

$$
\psi=\alpha \psi_{1}+\beta \psi_{2}+\gamma \psi_{3}+\delta \psi_{4}
$$

The eigenvalue-set $(\alpha, \beta, \gamma, \delta)$ determines also the eigenvectors of $G$ :

$$
\begin{aligned}
& \Phi_{1}=\frac{1}{\sqrt{2\left(1+\bar{k}^{2}\right)}}\left(1+\bar{k}^{2}, \mp \bar{k}, \pm 1,0\right) \\
& \Phi_{2}=\frac{1}{\sqrt{2\left(1+\bar{k}^{2}\right)}}\left(0, \pm 1, \pm \bar{k}, 1+\bar{k}^{2}\right)
\end{aligned}
$$

where $\bar{k}^{2}=k^{2}\left(n_{b_{2}}+1\right) /\left(n_{b_{1}}+1\right)$. Quantum-mechanical superpositions of these orthogonal eigenvectors with arbitrary (in general complex) weights $A \Phi_{1}+B \Phi_{2}$ lead to a density matrix over the Fermi algebra with eigenvalues

$$
\left(\frac{|A|^{2}}{2}, \frac{|B-\bar{k} A|^{2}}{2\left(1+\bar{k}^{2}\right)}, \frac{|A+\bar{k} B|^{2}}{2(1+\bar{k})^{2}}, \frac{|B|^{2}}{2}\right) .
$$

In order to maximize the entanglement we have to choose $A$ and $B$ such that $|A+\bar{k} B|=$ $|B-\bar{k} A|$, which is achieved for $A=i B$. With this, the entropy becomes $2 \ln 2$, so that 
the corresponding state is the tracial state over the Fermi algebra. To minimize the entanglement we have to make $|A+\bar{k} B|$ and $|B-\bar{k} A|$ as different as possible. This is guaranteed for both $A$ and $B$ real, e.g. for $A=\sin \varphi, B=\cos \varphi$. In Fig. 1 the dependence of the entanglement on the mixing parameter $\varphi$ and on the relative weight of the two components $\bar{k}$ is shown.
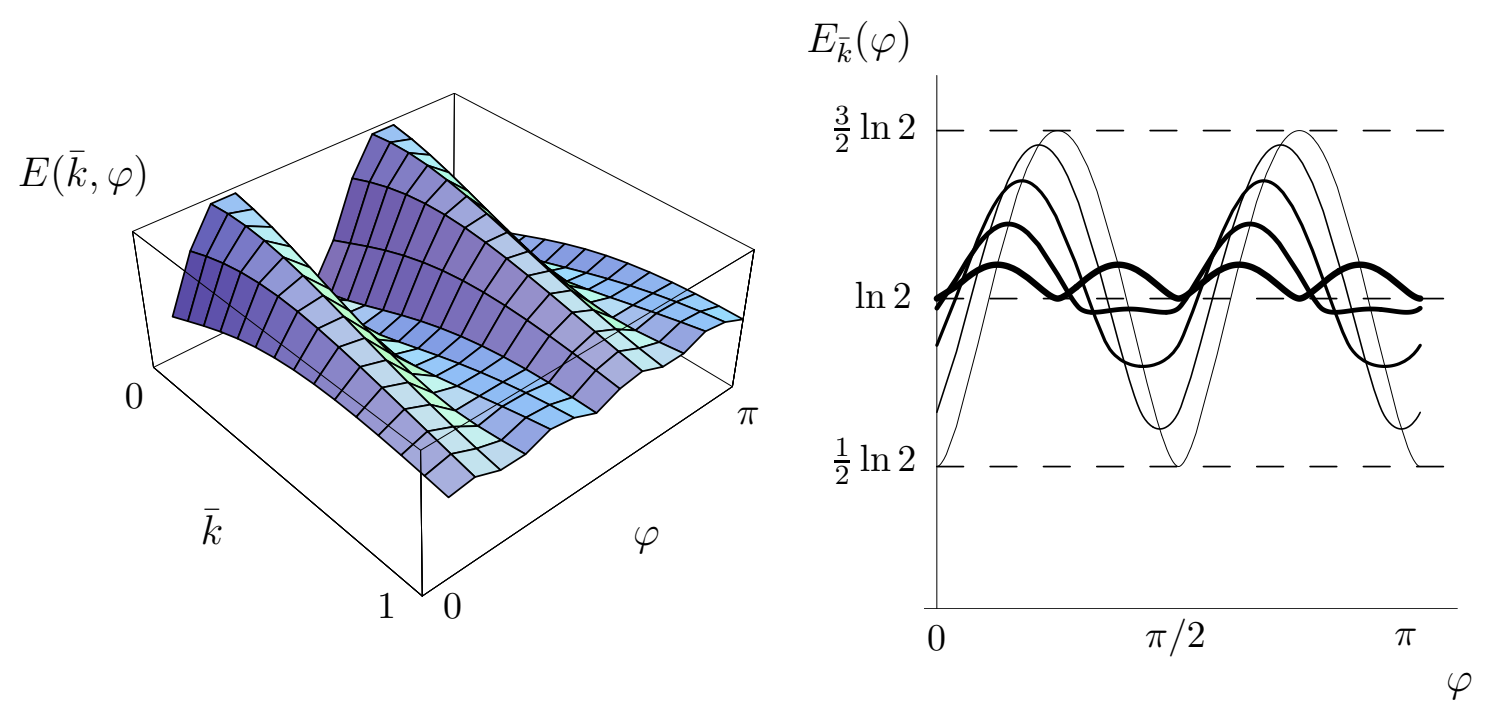

Figure 1: Entanglement of the "susino"-state: (a) in the complete parameter range; (b) for weight factors $\bar{k}=0,0.25,0.5,0.75,1$ (the line-thickness increases with $\bar{k}$ ).

The extremal points are then obtained by solving the equation

$$
\begin{aligned}
\frac{(\cos \varphi-\bar{k} \sin \varphi)(\sin \varphi+\bar{k} \cos \varphi)}{2\left(1+\bar{k}^{2}\right)} & \ln \frac{(\cos \varphi-\bar{k} \sin \varphi)^{2}}{(\sin \varphi+\bar{k} \cos \varphi)^{2}}- \\
& \sin \varphi \cos \varphi \ln \frac{\sin ^{2} \varphi}{\cos ^{2} \varphi}=0 .
\end{aligned}
$$

For $\bar{k}=0$ the minimum is achieved for $\sin \varphi=0$ or $\cos \varphi=0$ and amounts to $S(\rho)=$ $\ln 2$. The minimal entropy increases with $\bar{k}$ to reach for $\bar{k}=1$ its maximal value $3 / 2 \ln 2$, as is shown in Fig. 1b.

Fig. 2 shows the entropy (so, entanglement) of the first, resp. the second fermion of the susino states for a two-mode system. Zero entanglement occurs only for $\bar{k}=0$ when the second mode is not affected by the supertransformation. However, $E=0$ appears 

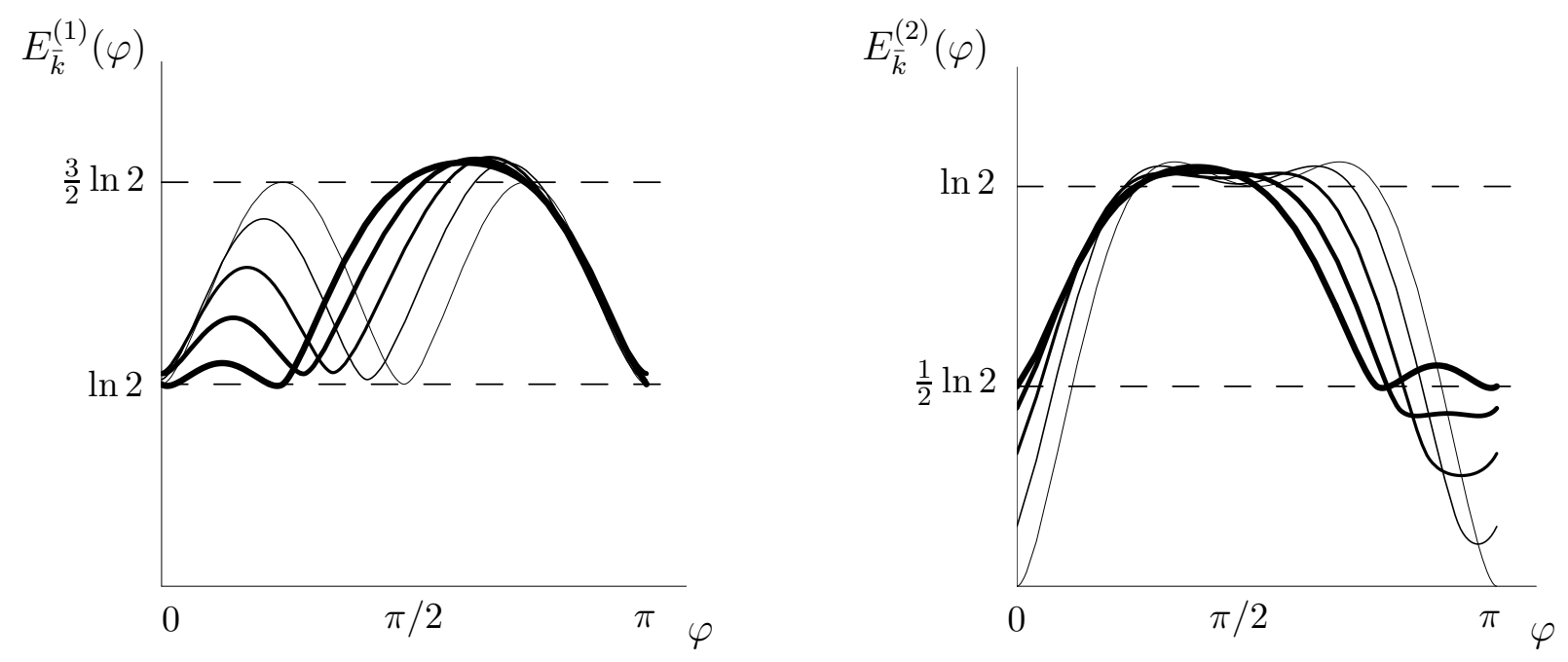

Figure 2: Entanglement of the first, resp. the second fermion for weight factors $\bar{k}=0,0.25,0.5,0.75,1$ (the line-thickness increases with $\bar{k}$ ).

only at two points, otherwise the mere existence of the other mode already influences the behavior of the system by creating some entanglement.

Thus for the entanglement of the eigenstates we find the following:

(i) The vacuum is not entangled, $E=0$;

(ii) The "one-mode" states $n_{b_{1}}=0$ and $n_{b_{2}}=0$ are characterized with $E=\ln 2$, however the entanglement of the first fermion w.r.t. the rest of the system is 0 while for the second fermion it is $\ln 2$ and vice versa;

(iii) In the general case the entanglement varies between its maximal value $E_{\max }=2 \ln 2$, which is independent on $\bar{k}$, and some minimal value $E_{\min }$ already depending on $\bar{k}$, for which $\ln 2 \leq E_{\min } \leq 3 / 2 \ln 2$.

\section{KMS-states}

A theorem due to Buchholz and Ojima [7] says that supersymmetry and KMS-structure are incompatible. More precisely, they show that an equilibrium state cannot be invariant under the evolution given by the odd derivations (1.8). Indeed, (with $x=e^{\beta}$, $\beta$ being the 
inverse temperature)

$$
\begin{aligned}
\omega\left(a^{\dagger} a\right) & =\frac{1}{1+x} & \omega\left(a a^{\dagger}\right) & =\frac{x}{1+x} \\
\omega\left(b^{\dagger} b\right) & =\frac{1}{x-1} & \omega\left(b b^{\dagger}\right) & =\frac{x}{x-1}
\end{aligned}
$$

is so different between bosons and fermions that it is hard to believe that this will not change by mixing them. Nevertheless we shall show that this happens miraculously if the evolution is governed by $e^{i G s}$. With the shorthand $(c, s)=(\cos s \sqrt{H}, \sin s \sqrt{H})$, this evolution reads

$$
a^{\dagger}(s) a(s)=a^{\dagger} a+i \frac{c s}{\sqrt{H}}\left[G, a^{\dagger} a\right]+\frac{s^{2} G}{H}\left[a^{\dagger} a, G\right] .
$$

We need consider only $\omega\left(a^{\dagger} a\right)$ since $a^{\dagger} a+b^{\dagger} b$ does not change and if one is invariant so is the other. Now

$$
\begin{aligned}
& {\left[a^{\dagger} a, G\right]=a^{\dagger} b-b^{\dagger} a} \\
& G\left[a^{\dagger} a, G\right]=a^{\dagger} b b^{\dagger} a-b^{\dagger} a a^{\dagger} b
\end{aligned}
$$

and

$$
\begin{aligned}
& \omega\left(\left[a^{\dagger} a, G\right]\right)=0 \\
& \omega\left(G\left[a^{\dagger} a, G\right]\right)=\frac{1}{x+1} \frac{x}{x-1}-\frac{x}{x+1} \frac{1}{x-1}=0 .
\end{aligned}
$$

$G$ is invariant under this supertransformation but $G_{\mathcal{A}}$ is not and we still have to verify that its thermal expectation remains zero. Indeed, with $\left[G, G_{\mathcal{A}}\right]=2 i\left(b^{\dagger} b-a^{\dagger} a-2 a^{\dagger} a b^{\dagger} b\right)$, this turns out to be true

$$
\omega\left(\left[G, G_{\mathcal{A}}\right]\right)=2 i\left(\frac{1}{x-1}-\frac{1}{x+1}-2 \frac{1}{x+1} \frac{1}{x-1}\right)=0 .
$$

So there remains the question of $c$ and $s$. These are functions given by convergent series of the form $\sum_{k=o}^{\infty} c_{k}\left(s^{2} H\right)^{k}$ and $\operatorname{Tr} \mathrm{e}^{-\beta \mathrm{H}} \mathrm{H}^{\mathrm{k}} \mathrm{A}=\partial^{\mathrm{k}} / \partial \beta^{\mathrm{k}} \operatorname{Tr} \mathrm{e}^{-\beta \mathrm{H}} \mathrm{A}$. But since the expectation values of the additional terms vanish for all $\beta$ the factors $c s / \sqrt{H}, s^{2} / \sqrt{H}$ do not change that and we conclude

$$
\omega\left(a^{\dagger}(s) a(s)\right)=\omega\left(a^{\dagger} a\right)=\frac{1}{1+x} .
$$

On the contrary with evolution (2.3), as we are dealing not with automorphisms but only with a one-parameter group of maps, we get for $Q=a^{\dagger} b$

$$
\omega(Q(s))=\omega(Q(0))+s \omega(H)=s \omega(H) \neq 0
$$

in agreement with the Buchholz-Ojima theorem. 


\section{Concluding remarks}

To summarize, we have studied four different transformations of three mixed Bose-Fermi algebras that do not respect the grading. In all four cases we have one-parameter groups of transformations which commute with the time evolution, generated by $H$. In Ia they are automorphisms, in Ib only linear and *-preserving maps, in II and III they do not transform $\mathcal{A}$ into $\mathcal{A}$. The case Ia represents an explicit form of a nonlinear transformation of creation and destruction operators which preserves the CCR/ CAR structure.

A state gives a representation in a Hilbert space and an associated probability interpretation, therefore an important question to be discussed is what happens under these transformations with the states. For the three algebras we get

I. The usual probabilities;

II. SUSY transforms into states of zero norm, so in all probabilities nothing happens;

III. With nonzero probability SUSY creates the Clifford object $\theta, \bar{\theta}$ which actually is unobservable.

We have identified the eigenvectors of the unitary implementer of the supertransformation as SUSY-quasiparticle states (susinos) with mixed statistics. Except for the vacuum, the susino-states are entangled. In the degeneracy space of $G$ the entanglement varies between the maximal possible value and some minimal value which is bigger than $\ln 2$.

Another natural question is the one about the invariant structures. Though SUSY mixes fermions with bosons, there should be combinations of them which remain invariant. They can be readily constructed. In fact, one can find a time evolution commuting with the SUSY transformation such that only these objects and not the bosons and the fermions are time-invariant. The situation is analogous to the $C$-breaking in the $K^{0}-\bar{K}^{0}$ system. Finally one can ask about SUSY-invariant states and in all cases the Fock vacuum provides such an example. Less trivial is the thermal distribution of bosons and fermions which

(i) is invariant under Ia;

(ii) is not invariant under Ib (the Buchholz-Ojima theorem [7]);

(iii) is trivially invariant under II;

(iv) is not invariant under III. 


\section{Acknowledgements}

We thank H. Grosse, E. Langmann, P. Minkowski, H. Nikolai and J. Wess for the discussions and D. Buchholz and R. Haag for valuable remarks. N.I. acknowledges the hospitality at the Erwin Schrödinger International Institute for Mathematical Physics, where part of this work has been completed.

\section{References}

[1] J. Wess, J. Bagger, Supersymmetry and Supergravity. 2nd ed. (Princeton Univ. Press, Princeton, NJ, 1992).

[2] E. Witten, Nucl. Phys. B185 (1981) 513-554.

[3] R.Y. Levine and Y. Tomozawa, J. Math. Phys. 23 (1982) 1415-1421.

[4] P. Freund, Introduction to Supersymmetry. (Cambridge Univ. Press, Cambridge, 1986).

[5] L. Girardello, M.T. Grisaru, P. Salomonson, Nucl. Phys. B178 (1981) 331-360.

[6] L. van Hove, Nucl. Phys. B207 (1982) 15-28.

[7] D. Buchholz, I. Ojima, Nucl. Phys. B498 (1997) 228-242.

[8] H. Grosse, L. Pittner, J. Math. Phys. 29 (1988) 110-118.

[9] H. Grosse, E. Langmann, Phys. Lett. A176 (1993) 307-312.

[10] D. Buchholz, in: Lecture Notes in Physics, vol. 539 (2000) 211-220 (hep-th/ 9812179).

[11] N. Seiberg, JHEP 0306 (2003) 010 (hep-th/0305248).

[12] J. Wess, B. Zumino, Nucl. Phys. B70 (1974) 39-50.

[13] A. Jaffe, A. Lesniewski, and J. Weitsman, Commun. Math. Phys. 112 (1987) 75-88.

[14] H. Nicolai, J. Math. A: Math. Gen. 9 (1976) 1497-1506. 